\title{
Author Correction: Network analysis of multivariate data in psychological science
}

\author{
Denny Borsboom (iD, Marie K. Deserno, Mijke Rhemtulla, Sacha Epskamp, Eiko I. Fried, Richard J. McNally, \\ Donald J. Robinaugh, Marco Perugini (D), Jonas Dalege, Giulio Constantini, Adela-Maria Isvoranu (D), Anna C. Wysocki, \\ Claudia D. van Borkulo, Riet van Bork (D) and Lourens J. Waldorp
}

Correction to: Nature Reviews Methods Primers (2021) https://doi.org/10.1038/s43586-021-00055-w, published online 19 August 2021.

In the original version of the article, the node labels in Figure 4 were incorrectly represented, and Figure 8 was inaccurate due to a coding error. In the legend of Figure $7 \mathrm{~b}$, the grey squares and dark squares refer to the value zero and not, respectively. These errors have been corrected in the HTML and PDF versions of the article and the original versions of Figures 4 and 8 are shown below.

https://doi.org/10.1038/s43586-022-00101-1। Published online: 21 February 2022

(c) Springer Nature Limited 2022
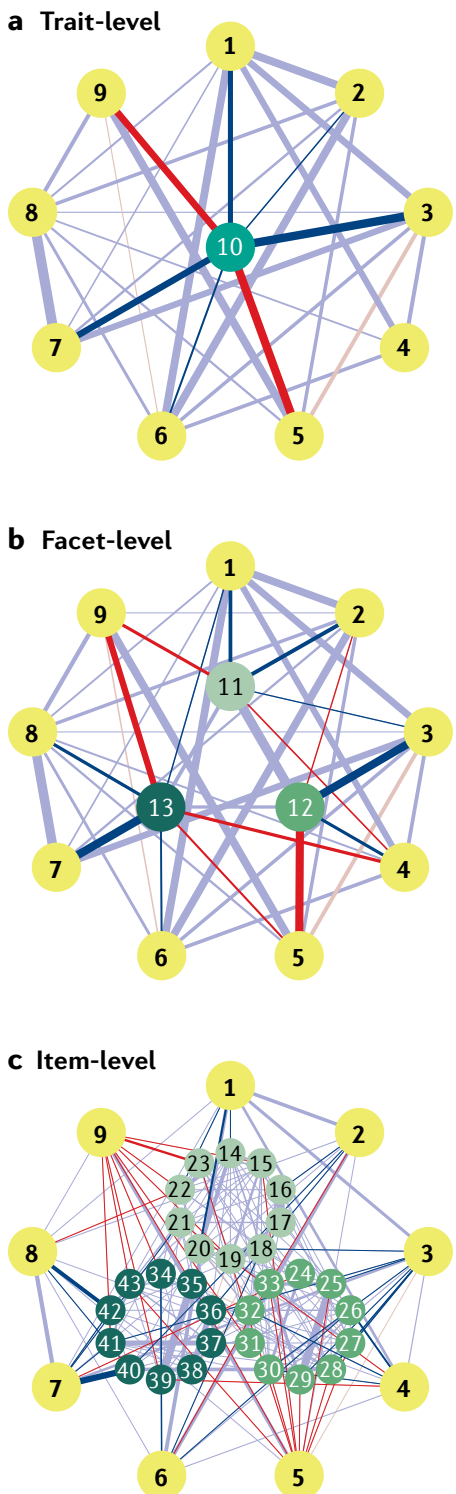

d Centrality of goals

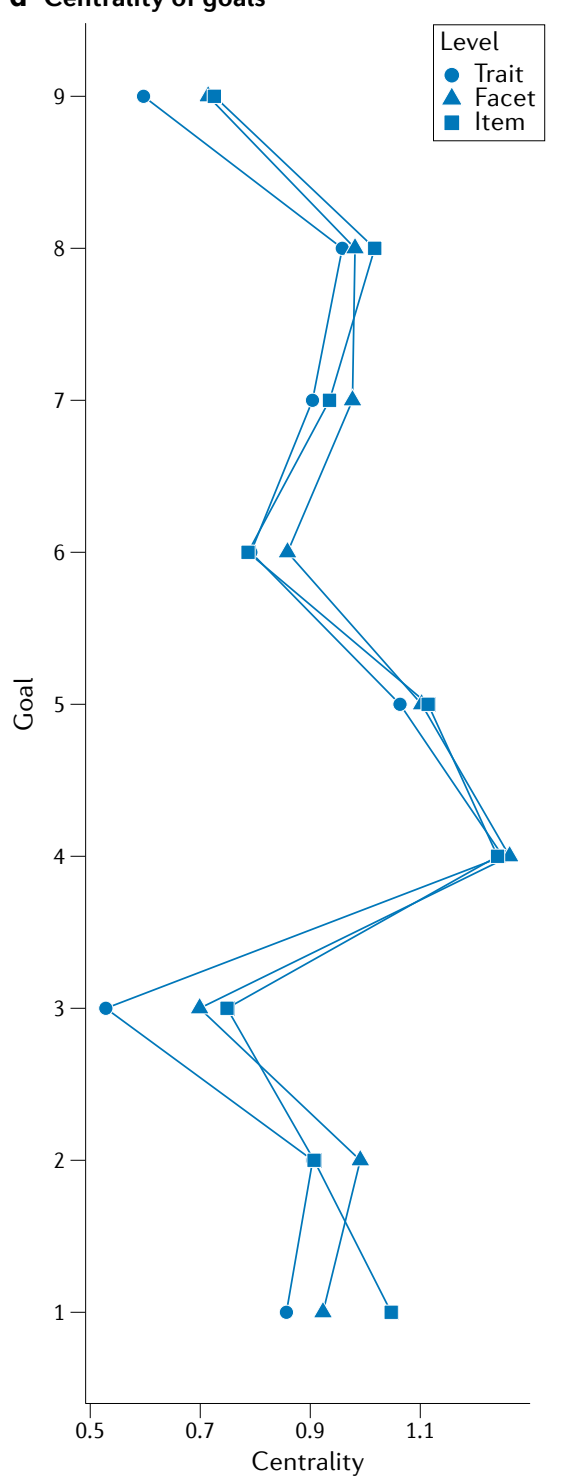

Goals

1: Comply with rules

2: Have control

3: Personal realization

4: Do something well, avoid mistakes

5: Accomplish something, observe a commitment

6: Avoid or manage things you do not care about

7: Be safe

8: Think, reflect

9: Do not think

Trait

10: Conscientiousness

Facets

11: Orderliness

12: Industriousness

13: Impulse-control

Items

14: Precise

15: Imprecise*

16: Ordered

17: Disordered*

18: Organized

19: Disorganized*

20: Careful

21: Chaotic*

22: Fussy

23: Haphazard*

24: Industrious

25: Layabout*

26: Effective

27: Erratic*

28: Diligent

29: Unwilling*

30: Hardworking

31: Negligent*

32: Tenacious

33: Lazy*

34: Reflective

35: Instinctive*

36: Controlled

37: Reckless*

38: Cautious

39: Impulsive*

40: Disciplined

41: Profligate*

42: Prudent

43: Imprudent*

Fig. 4 | Strength centrality estimates for all nodes in three networks of personality research data. 
CORRECTIONS

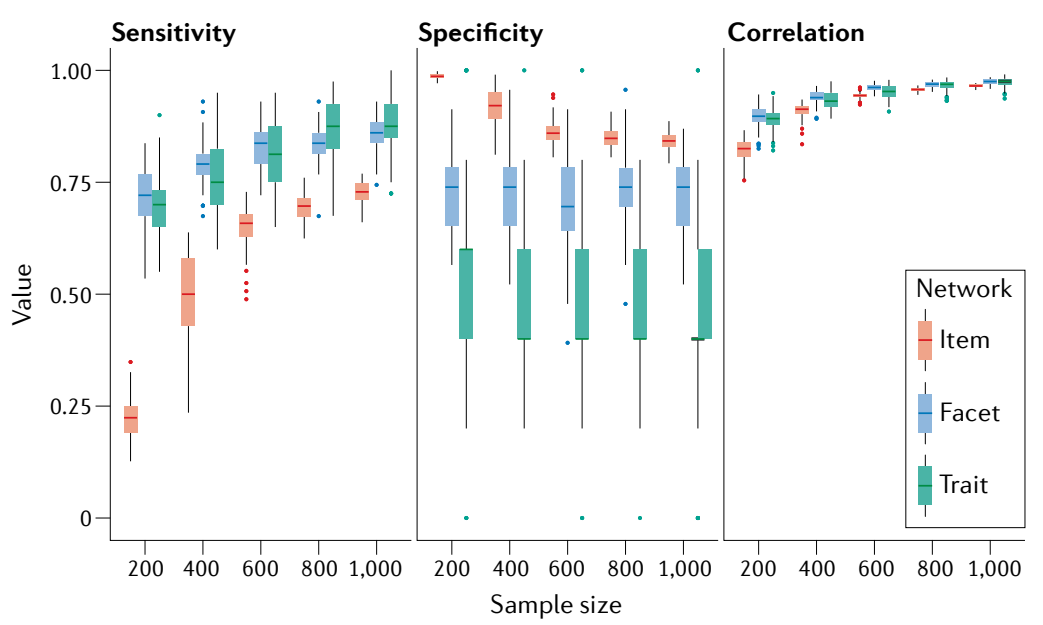

Fig. 8 | Projected replicability for the personality network as assessed through the ReplicationSimulator function in the bootnet $R$ package. 